\title{
On Boundedness and Attractiveness of Nonlinear Switched Delay Systems
}

\author{
Yi Zhang, ${ }^{1}$ Yuyun Zhao, ${ }^{1}$ Honglei Xu, ${ }^{2,3}$ Hongting Shi, ${ }^{4}$ and Kok Lay Teo ${ }^{3}$ \\ ${ }^{1}$ Department of Mathematics, China University of Petroleum (Beijing), Beijing 102249, China \\ ${ }^{2}$ School of Energy and Power Engineering, Huazhong University of Science and Technology, Wuhan 430074, China \\ ${ }^{3}$ Department of Mathematics and Statistics, Curtin University, Perth, WA 6845, Australia \\ ${ }^{4}$ School of Mathematical Science, Capital Normal University, Beijing 1000484, China
}

Correspondence should be addressed to Honglei Xu; h.xu@curtin.edu.au and Hongting Shi; shihongting302@163.com

Received 1 August 2013; Accepted 29 October 2013

Academic Editor: Yong Hong Wu

Copyright (C) 2013 Yi Zhang et al. This is an open access article distributed under the Creative Commons Attribution License, which permits unrestricted use, distribution, and reproduction in any medium, provided the original work is properly cited.

\begin{abstract}
This paper is concerned with the boundedness and attractiveness of nonlinear switched delay systems whose subsystems have different equilibria. Some sufficient conditions which can guarantee the system's boundedness are obtained. In addition, we work out the region where the solution will remain and furthermore the relationship between the initial function and the bounded region. Based on the new concept of attractor with switching laws, we show that the nonlinear switched delay system is attractive and then obtain the attractive region.
\end{abstract}

\section{Introduction}

A switched system is a collection of finite continuous variable systems (called subsystems) along with a discrete event governing the "switching" among them (called the switching law). In reality, there are many switched systems that occur naturally or by design, such as those in the fields of control, communication, computer, and signal processes. Indeed, these systems are always suited to describe practical dynamical behaviors with hybrid nature in engineering and technology $[1,2]$. In the last decades, they have attracted considerable attention among control theorists, computer scientists, and practicing engineers in the study of switched systems and switching control design and hence rich theoretical results have been obtained (see [3-20] and the references therein).

Up to now, most of the studies on switched systems require that all subsystems share a common equilibrium. However, in many real world problems, the assumption that all subsystems share a common equilibrium may not hold and thus the assumption may limit the applicability of stability results. Recently, it is pointed out in $[21,22]$ that, when subsystems have different equilibria or no equilibrium, a switched system can still exhibit interesting behaviors under appropriate switching laws. Such behaviors are similar to those of a conventional bounded or stable system near an equilibrium point. In this paper, we introduce some boundedness and attractiveness notions to define such behaviors for switched delay systems. Such notions are extensions of the traditional boundedness and attractiveness concepts in $[15,16]$.

In this paper, we focus on the boundedness and attractiveness problems for a simple yet important class of nonlinear switched delay systems, which includes three main contributions. First, we propose the concept of attractor with the switching law and the notation of a function $\varphi \in C_{\tau}$ in the neighbourhood of a subset $\Omega \in R^{n}$ which is different from the conventional concept of the distance between two subsets of $R^{n}$. Second, we propose sufficient conditions which guarantee that the switched delay system is bounded or attractive. Third, we explicitly construct a switching law and the region in which the solutions remain (boundedness) and explicitly construct the attractor for the switched delay systems. 


\section{Problem Statement}

Consider the nonlinear switched delay system

$$
\dot{x}=A_{\gamma} x+f_{\gamma}(x(t-\tau)), \quad \gamma \in \mathbb{\sharp} \triangleq\{1,20, \ldots, N\},
$$

where $x \in R^{n}, f_{\gamma} \in C\left(R^{n}, R^{n}\right)$, and $A_{\gamma} \in R^{n \times n}, \tau \geq 0$. It is assumed that

(H1) there exist $\alpha>0, M \geq 1$ such that $\left\|e^{A_{\gamma} t}\right\| \leq M e^{-\alpha t}$;

(H2) there exists $L, 0<L<\alpha / M$, such that $\| f_{\gamma}(x)-$ $f_{\gamma}(y)\|\leq L\| x-y \|, x, y \in \mathbb{R}^{n}, \gamma \in \llbracket ;$

(H3) $A_{\gamma} x+f_{\gamma}(x)=0$ has only one solution $x^{\gamma} \in \mathbb{R}^{n}$ and $x^{\gamma} \neq x^{l}$ for $\gamma \neq l, \gamma, l \in \mathbb{\square}$.

Let $\Gamma=\left\{x_{\gamma}\left(t ; 0, x^{j}\right), \gamma, j \in \mathbb{Q}, t \in R^{+}\right\}$, where

$$
x_{\gamma}\left(t ; 0, x^{j}\right)=e^{A_{\gamma} t} x^{j}+\int_{0}^{t} e^{A_{\gamma}(t-u)} f_{\gamma}\left(x_{\gamma}(u-\tau)\right) d u
$$

and denote that $\widetilde{D}=\max \left\{\left\|x^{\gamma}-x^{j}\right\|, \gamma \neq j, \gamma, j \in \mathbb{Q}\right\}, C_{\tau} \triangleq$ $C\left([-\tau, 0], R^{n}\right)$, and for $\varphi \in C_{\tau}$, define

$$
\|\varphi\|_{\tau}=\sup _{\theta \in[-\tau, 0]}\|\varphi(\theta)\| .
$$

Let $x_{\gamma}\left(t ; t_{0}, \varphi\right) \triangleq x_{\gamma}(t)$ be the solution of the initial value problem

$$
\begin{gathered}
\dot{x}_{\gamma}(t)=A_{\gamma} x_{\gamma}+f_{\gamma}\left(x_{\gamma}(t-\tau)\right), \\
x_{\gamma}(\theta)=\varphi(\theta), \quad \theta \in[-\tau, 0] .
\end{gathered}
$$

If $\varphi \equiv x^{j}$ especially, denote that $x_{\gamma}\left(t ; t_{0}, x^{j}\right) \triangleq \tilde{x}_{\gamma}^{j}(t), j \in$ q, and let $x\left(t ; t_{0}, \varphi, \sigma\right) \triangleq x(t)$ be the solution of (1) with the switching law $\sigma$ as

$$
\begin{gathered}
\dot{x}(t)=A_{\gamma} x+f_{\gamma}(x(t-\tau)), \quad \gamma \in \mathbb{\mathbb { V }}, \\
x(\theta)=\varphi(\theta), \quad \theta \in[-\tau, 0],
\end{gathered}
$$

where $x_{t} \triangleq x(t+\theta), \theta \in[-\tau, 0]$. Since all the subsystems are autonomous, we can take $t_{0}=0$ without loss of generality. Let $\sigma=\left(\left(t_{1}, i_{1}\right), \ldots,\left(t_{k}, i_{k}\right), \ldots\right)$ be the switching law of the switched system (1), where $i_{k} \in \mathbb{\square}$ and $0=t_{0}<t_{1}<\cdots<$ $t_{k}<\cdots$; that is, under the switching law $\sigma$, the subsystem $i_{k}$ is active during the time interval $\left[t_{k-1}, t_{k}\right)$. Suppose that $i_{k} \neq i_{k+1}$ holds, which means that a subsystem will be definitely switched to a different subsystem when the switching triggers.

\section{Boundedness}

In this section, we will study the boundedness problem of the switched delay system (1).

Definition 1. Suppose that $\delta>0, \Omega \subset R^{n}$, and then a neighborhood of $\Omega$ in $C_{\tau}$ is defined as

$$
B_{\delta}(\Omega)=\left\{\varphi \mid \varphi \in C_{\tau}, \sup _{\theta \in[-\tau, 0]} \rho(\varphi(\theta), \Omega) \leq \delta\right\},
$$

where the distance $\rho$ between $x \in R^{n}$ and $\Omega$ is defined as

$$
\rho(x, \Omega)=\inf \{\|x-y\| \mid y \in \Omega\} .
$$

Definition 2. A nonnegative number $\mu$ is called the dwell time of the switching law

$$
\sigma=\left(\left(t_{1}, i_{1}\right), \ldots,\left(t_{k}, i_{k}\right), \ldots\right)
$$

if

$$
\mu=\inf \left\{\left|t_{k+1}-t_{k}\right|, k=1,2, \ldots\right\}
$$

holds.

Lemma 3. Let $\mu$ be the dwell time of the switching law $\sigma=$ $\left(\left(t_{1}, i_{1}\right), \ldots,\left(t_{k}, i_{k}\right), \ldots\right)$, and $\delta>0$ is a constant. If $\mu \geq$ $\max \{\tau,(\ln (M(\delta+\widetilde{D}) / \delta)) /(\alpha-M L)\}$, then $\varphi \in B_{\delta}\left(x^{i_{0}}\right)$ implies that $x_{t_{k}} \in B_{\delta}\left(x^{i_{k}}\right)$, where

$$
x_{t_{k}}=x_{t_{k}}(\theta)=x\left(t_{k}+\theta, 0, \varphi, \sigma\right), \quad \theta \in[-\tau, 0] .
$$

Proof. Suppose that $\varphi \in B_{\delta}\left(x^{i_{0}}\right)$ and the switching law is defined as $\sigma=\left(\left(t_{1}, i_{1}\right), \ldots,\left(t_{k}, i_{k}\right), \ldots\right)$. Then, we will use induction to prove the result. When $m=1, t \in\left[0, t_{1}\right]$, we will prove that

$$
\sup _{\theta \in[-\tau, 0]}\left\|x\left(t_{1}+\theta, 0, \varphi, \sigma\right)-x^{i_{1}}\right\| \leq \delta .
$$

In fact,

$$
\begin{aligned}
& \left\|x(t, 0, \varphi, \sigma)-x^{i_{1}}\right\| \\
& =\left\|x_{i_{1}}(t)-x^{i_{1}}\right\| \\
& =\| e^{A_{i_{1}} t} \varphi(0)+\int_{0}^{t} e^{A_{i_{1}}(t-u)} f_{i_{1}}\left(x_{i_{1}}(u-\tau)\right) d u \\
& -e^{A_{i_{1}} t} x^{i_{1}}-\int_{0}^{t} e^{A_{i_{1}}(t-u)} f_{i_{1}}\left(x^{i_{1}}\right) d u \| \\
& \leq\left\|e^{A_{i_{1}} t}\right\|\left\|\varphi(0)-x^{i_{1}}\right\| \\
& +\int_{0}^{t}\left\|e^{A_{i_{1}}(t-u)}\right\|\left\|f_{i_{1}}\left(x_{i_{1}}(u-\tau)\right)-f_{i_{1}}\left(x^{i_{1}}\right)\right\| d u \\
& \leq M e^{-\alpha t}\left\|\varphi(0)-x^{i_{1}}\right\| \\
& +M L \int_{0}^{t} e^{-\alpha(t-u)}\left\|x_{i_{1}}(u-\tau)-x^{i_{1}}\right\| d u .
\end{aligned}
$$


Abstract and Applied Analysis

3

Then,

$$
\begin{aligned}
& e^{\alpha t}\left\|x_{i_{1}}(t)-x^{i_{1}}\right\| \\
& \leq M\left\|\varphi(0)-x^{i_{1}}\right\|+M L \int_{0}^{t} e^{\alpha u}\left\|x_{i_{1}}(u-\tau)-x^{i_{1}}\right\| d u \\
& \leq M\left\|\varphi(0)-x^{i_{1}}\right\|+M L \int_{0}^{t} e^{\alpha u}\left\|x_{i_{1}}(u-\tau)-x^{i_{1}}\right\| d u \\
& \leq M\left[\left\|\varphi(0)-x^{i_{0}}\right\|+\left\|x^{i_{0}}-x^{i_{1}}\right\|\right] \\
& \quad+M L \int_{0}^{t} e^{\alpha u}\left\|x_{i_{1}}(u-\tau)-x^{i_{1}}\right\| d u \\
& \leq M[\delta+\widetilde{D}]+M L \int_{0}^{t} e^{\alpha u}\left\|x_{i_{1} u}-x^{i_{1}}\right\|_{\tau} d u .
\end{aligned}
$$

Furthermore, since the right hand side of the inequality is increasing function, we have

$$
\begin{aligned}
e^{\alpha t}\left\|x_{i_{1} t}-x^{i_{1}}\right\|_{\tau} \leq & M[\delta+\widetilde{D}] \\
& +M L \int_{0}^{t} e^{\alpha u}\left\|x_{i_{1} u}-x^{i_{1}}\right\|_{\tau} d u,
\end{aligned}
$$

and then, by applying the Bellman-Gronwall inequality to (14), we have

$$
e^{\alpha t}\left\|x_{i_{1} t}-x^{i_{1}}\right\|_{\tau} \leq M[\delta+\widetilde{D}] e^{M L t}
$$

and thus

$$
\left\|x_{i_{1} t}-x^{i_{1}}\right\|_{\tau} \leq M[\delta+\widetilde{D}] e^{-(\alpha-M L) t} .
$$

Let $t=t_{1}$; we get

$$
\left\|x_{t_{1}}-x^{i_{1}}\right\|_{\tau}=\left\|x_{i_{1} t_{1}}-x^{i_{1}}\right\|_{\tau} \leq M[\delta+\widetilde{D}] e^{-(\alpha-M L) t_{1}} \leq \delta,
$$

and (11) is proved. Suppose that, when $m=k$, the result is right; that is, $x_{t_{k}} \in B_{\delta}\left(x^{i_{k}}\right)$, and then we will prove that $x_{t_{k+1}} \in$ $B_{\delta}\left(x^{i_{k+1}}\right)$; that is,

$$
\sup _{\theta \in[-\tau, 0]}\left\|x\left(t_{k+1}+\theta, 0, \varphi, \sigma\right)-x^{i_{k+1}}\right\| \leq \delta
$$

In fact, when $t \in\left[t_{k}, t_{k+1}\right], x(t, 0, \varphi, \sigma)=x_{i_{k+1}}\left(t-t_{k}, 0, x_{t_{k}}\right)$, and thus

$$
\begin{aligned}
& \left\|x(t, 0, \varphi, \sigma)-x^{i_{k+1}}\right\| \\
& =\left\|x_{i_{k+1}}\left(t-t_{k}, 0, x_{t_{k}}\right)-x^{i_{k+1}}\right\| \\
& =\| e^{A_{i_{k+1}}\left(t-t_{k}\right)} x_{t_{k}}(0) \\
& +\int_{0}^{t-t_{k}} e^{A_{i_{k+1}}\left(t-t_{k}-u\right)} f_{i_{k+1}}\left(x_{i_{k+1}}(u-\tau)\right) d u \\
& -e^{A_{i_{k+1}}\left(t-t_{k}\right)} x^{i_{k+1}} \\
& -\int_{0}^{t-t_{k}} e^{A_{i_{k+1}}\left(t-t_{k}-u\right)} f_{i_{k+1}}\left(x^{i_{k+1}}\right) d u \| \\
& \leq\left\|e^{A_{i_{k+1}}\left(t-t_{k}\right)}\right\|\left\|x_{t_{k}}(0)-x^{i_{k+1}}\right\| \\
& +\int_{0}^{t-t_{k}}\left\|e^{A_{i_{k+1}}\left(t-t_{k}-u\right)}\right\| \\
& \times\left\|f_{i_{k+1}}\left(x_{i_{k+1}}(u-\tau)\right)-f_{i_{k+1}}\left(x^{i_{k+1}}\right)\right\| d u \\
& \leq M e^{-\alpha\left(t-t_{k}\right)}\left\|x_{t_{k}}(0)-x^{i_{k+1}}\right\| \\
& +M L \int_{0}^{t-t_{k}} e^{-\alpha\left(t-t_{k}-u\right)}\left\|x_{i_{k+1}}(u-\tau)-x^{i_{k+1}}\right\| d u,
\end{aligned}
$$

and then we have

$$
\begin{aligned}
& e^{\alpha\left(t-t_{k}\right)}\left\|x_{i_{k+1}}\left(t-t_{k}, 0, \varphi\right)-x^{i_{k+1}}\right\| \\
& \leq M\left\|x_{t_{k}}(0)-x^{i_{k+1}}\right\| \\
& \quad+M L \int_{0}^{t-t_{k}} e^{\alpha u}\left\|x_{i_{k+1}}(u-\tau)-x^{i_{k+1}}\right\| d u \\
& \leq M\left[\left\|x_{t_{k}}(0)-x^{i_{k}}\right\|+\left\|x^{i_{k}}-x^{i_{k+1}}\right\|\right] \\
& \quad+M L \int_{0}^{t-t_{k}} e^{\alpha u}\left\|x_{i_{k+1}}(u-\tau)-x^{i_{k+1}}\right\| d u \\
& \leq M[\delta+\widetilde{D}]+M L \int_{0}^{t-t_{k}} e^{\alpha u}\left\|x_{i_{k+1} u}-x^{i_{k+1}}\right\|_{\tau} d u .
\end{aligned}
$$

Since the right hand side of the inequality is increasing function, we have

$$
\begin{aligned}
& e^{\alpha\left(t-t_{k}\right)}\left\|x_{i_{k+1}\left(t-t_{k}\right)}-x^{i_{k+1}}\right\|_{\tau} \\
& \quad \leq M[\delta+\widetilde{D}]+M L \int_{0}^{t-t_{k}} e^{\alpha u}\left\|x_{i_{k+1} u}-x^{i_{k+1}}\right\|_{\tau} d u .
\end{aligned}
$$

Applying the Bellman-Gronwall inequality to (21) yields

$$
\begin{aligned}
\left\|x_{i_{k+1}\left(t-t_{k}\right)}-x^{i_{k+1}}\right\|_{\tau} & =\left\|x_{t}-x^{i_{k+1}}\right\|_{\tau} \\
& \leq M[\delta+\widetilde{D}] e^{-(\alpha-M L)\left(t-t_{k}\right)} .
\end{aligned}
$$


Let $t=t_{k+1}$, and then we get

$$
\left\|x_{t_{k+1}}-x^{i_{k+1}}\right\|_{\tau} \leq M[\delta+\widetilde{D}] e^{-(\alpha-M L)\left(t_{k+1}-t_{k}\right)} \leq \delta,
$$

and (18) is proved. The proof is completed.

Remark 4. Lemma 3 shows that if the initial function $\varphi$ is in the $\delta$ neighborhood of $x^{i_{0}}$, then the solution $x(t, 0, \varphi, \sigma)$ with the switching law $\sigma$ whose dwell time satisfies $\mu \geq$ $\max \{\tau,(\ln (M(\delta+\widetilde{D}) / \delta)) /(\alpha-M L)\}$ has the following property: at the $k$ th switching point, the function $x\left(t_{k}+\right.$ $\theta, 0, \varphi, \sigma)(\theta \in[-\tau, 0])$ is in the $\delta$ neighborhood of $x^{i_{k}}$.

Theorem 5. Let $\mu$ be the dwell time of the switching law $\sigma=\left(\left(t_{1}, i_{1}\right), \ldots,\left(t_{k}, i_{k}\right), \ldots\right)$ and $\delta>0$ is a constant. If $\mu \geq$ $\max \{\tau, \ln (M(\delta+\widetilde{D}) / \delta) /(\alpha-M L)\}$, then $\varphi \in B_{\delta}\left(x^{i_{0}}\right)$ implies that the solution $x(t, 0, \varphi, \sigma)$ of (1) satisfies $x(t) \in B_{M \delta}(\Gamma)$.

Proof. We use mathematical induction to prove the result. When $t \in\left[0, t_{1}\right]$,

$$
\begin{aligned}
& \rho(x(t, 0, \varphi, \sigma), \Gamma) \\
& \leq\left\|x(t, 0, \varphi, \sigma)-x\left(t, 0, x^{i_{0}}, \sigma\right)\right\| \\
& =\left\|x_{i_{1}}(t, 0, \varphi, \sigma)-\tilde{x}_{i_{1}}\left(t, 0, x^{i_{0}}\right)\right\| \\
& =\left\|x_{i_{1}}(t)-\tilde{x}_{i_{1}}(t)\right\| \\
& =\| e^{A_{i_{1}} t} \varphi(0)+\int_{0}^{t} e^{A_{i_{1}}(t-u)} f_{i_{1}}\left(x_{i_{1}}(u-\tau)\right) d u \\
& -e^{A_{i_{1}} t} x^{i_{0}}-\int_{0}^{t} e^{A_{i_{1}}(t-u)} f_{i_{1}}\left(\tilde{x}_{i_{1}}(u-\tau)\right) d u \| \\
& \leq\left\|e^{A_{i_{1}} t}\left(\varphi(0)-x^{i_{0}}\right)\right\| \\
& +\left\|\int_{0}^{t} e^{A_{i_{1}}(t-u)}\left(f_{i_{1}}\left(x_{i_{1}}(u-\tau)\right)-f_{i_{1}}\left(\tilde{x}_{i_{1}}(u-\tau)\right)\right) d u\right\| \\
& \leq M e^{-\alpha t}\left\|\varphi(0)-x^{i_{0}}\right\| \\
& +L M \int_{0}^{t} e^{-\alpha(t-u)}\left\|x_{i_{1}}(u-\tau)-\tilde{x}_{i_{1}}(u-\tau)\right\| d u \\
& \leq e^{-\alpha t}\left[M \delta+L M \int_{0}^{t} e^{\alpha u}\left\|x_{i_{1} u}-\tilde{x}_{i_{1} u}\right\|_{\tau} d u\right],
\end{aligned}
$$

which implies that

$$
\begin{gathered}
e^{\alpha t}\left\|x_{i_{1} t}-\tilde{x}_{i_{1} t}\right\|_{\tau} \leq M \delta+L M \int_{0}^{t} e^{\alpha u}\left\|x_{i_{1} u}-\tilde{x}_{i_{1} u}\right\|_{\tau} d u, \\
\left\|x_{i_{1} t}-\tilde{x}_{i_{1} t}\right\|_{\tau} \leq M \delta e^{-(\alpha-L M) t} \leq M \delta .
\end{gathered}
$$

Thus, for $t \in\left[0, t_{1}\right]$ and $\varphi \in B_{\delta}$,

$$
\rho(x(t, 0, \varphi, \sigma), \Gamma) \leq M \delta .
$$

Suppose that, when $t \in\left[t_{k-1}, t_{k}\right],(26)$ holds. Now we prove that, when $t \in\left[t_{k}, t_{k+1}\right]$,

$$
\rho(x(t, 0, \varphi, \sigma), \Gamma) \leq M \delta .
$$

In fact, by Lemma 3, we know that $x_{t_{k}} \in B_{\delta}\left(x^{i_{k}}\right)$ and

$$
\begin{aligned}
& \rho(x(t, 0, \varphi, \sigma), \Gamma) \\
& \leq\left\|x(t, 0, \varphi, \sigma)-\tilde{x}_{i_{k+1}}^{i_{k}}\left(t-t_{k}, 0, x^{i_{k}}\right)\right\| \\
& =\left\|x_{i_{k+1}}\left(t-t_{k}, 0, x_{t_{k}}, \sigma\right)-\tilde{x}_{i_{k+1}}^{i_{k}}\left(t-t_{k}, 0, x^{i_{k}}\right)\right\| \\
& =\left\|x_{i_{k+1}}\left(t-t_{k}\right)-\tilde{x}_{i_{k+1}}^{i_{k}}\left(t-t_{k}\right)\right\| \\
& =\| e^{A_{i_{k+1}}\left(t-t_{k}\right)} x_{t_{k}}(0) \\
& +\int_{0}^{t-t_{k}} e^{A_{i_{k+1}}\left(t-t_{k}-u\right)} f_{i_{k+1}}\left(x_{i_{k+1}}(u-\tau)\right) d u \\
& -e^{A_{i_{k+1}}\left(t-t_{k}\right)} x^{i_{k}} \\
& -\int_{0}^{t-t_{k}} e^{A_{i_{k+1}}\left(t-t_{k}-u\right)} f_{i_{k+1}}\left(\widetilde{x}_{i_{k+1}}^{i_{k}}(u-\tau)\right) d u \| \\
& \leq M e^{-\alpha\left(t-t_{k}\right)}\left\|x_{t_{k}}(0)-x^{i_{k}}\right\| \\
& +L M \int_{0}^{t-t_{k}} e^{-\alpha\left(t-t_{k}-u\right)}\left\|x_{i_{k+1}}(u-\tau)-\tilde{x}_{i_{k+1}}^{i_{k}}(u-\tau)\right\| d u \\
& \leq e^{-\alpha\left(t-t_{k}\right)}\left[M \delta+L M \int_{0}^{t-t_{k}} e^{\alpha u}\left\|x_{i_{k+1} u}-\tilde{x}_{i_{k+1}}^{i_{k}} u\right\|_{\tau} d u\right],
\end{aligned}
$$

which implies that

$$
\left\|x_{i_{k+1}}\left(t-t_{k}\right)-\tilde{x}_{i_{k+1}}^{i_{k}}\left(t-t_{k}\right)\right\|_{\tau} \leq M \delta e^{-(\alpha-L M)\left(t-t_{k}\right)} \leq M \delta
$$

and thus

$$
\rho(x(t, 0, \varphi, \sigma), \Gamma) \leq\left\|x_{i_{k+1}}\left(t-t_{k}\right)-\tilde{x}_{i_{k+1}}^{i_{k}}\left(t-t_{k}\right)\right\|_{\tau} \leq M \delta .
$$

Thus, for all $t \geq 0$, we have

$$
\rho(x(t, 0, \varphi, \sigma), \Gamma) \leq M \delta .
$$

The proof is completed.

Remark 6. (i) The result in Theorem 5 shows that if the initial function $\varphi$ is in the $\delta$ neighborhood of $x^{i_{0}}$, then the solution $x(t, 0, \varphi, \sigma)$ with the switching law whose dwell time satisfies $\mu \geq \max \{\tau,(\ln (M(\delta+\widetilde{D}) / \delta)) /(\alpha-M L)\}$ is in the $M \delta$ neighborhood of the set $\Gamma$.

(ii) Theorem 5 not only shows the boundedness of the switched system but also gives the region in which the solution will remain. 
By the same procedure of Lemma 3 and Theorem 5, we can study the boundedness of the solution of the following nonlinear switched multidelays system:

$$
\dot{x}=A_{\gamma} x+\sum_{j=1}^{P} f_{\gamma j}\left(x, x\left(t-\tau_{\gamma j}\right)\right), \quad \gamma \in \mathbb{\square} \triangleq\{1,2, \ldots, N\},
$$

where $x \in R^{n}, f_{\gamma j} \in C\left(R^{n} \times R^{n}, R^{n}\right), A_{\gamma} \in R^{n \times n}$, and $0 \leq \tau_{\gamma j} \leq$ $\tau$.

Assume that

(H1)' there exist $\alpha>0, M \geq 1$ such that $\left\|e^{A_{\gamma} t}\right\| \leq M e^{-\alpha t}$;

(H2)' there exists $L, 0<L<\alpha / 2 P M$, such that $\| f_{\gamma j}\left(x_{1}, x_{2}\right)-$ $f_{\gamma j}\left(y_{1}, y_{2}\right) \| \leq L\left[\left\|x_{1}-y_{1}\right\|+\left\|x_{2}-y_{2}\right\|\right], x_{1}, x_{2}, y_{1}, y_{2} \in$ $R^{n}, \gamma \in \mathbb{\square}$, and $j=1,2, \ldots, P$;

(H3)' $A_{\gamma} x+\sum_{j=1}^{P} f_{\gamma j}(x, x)=0$ has only one solution $x^{\gamma} \in$ $R^{n}$ and $x^{j} \neq x^{l}$ for $j \neq l, j, l \in \mathbb{\square}$.

Let $\Gamma^{\prime}=\left\{\widetilde{x}_{i}^{j}\left(t ; 0, x^{j}\right), i, j \in \mathbb{\square}, t \in R^{+}\right\}$. Similarly, we can obtain the following.

Lemma 7. Let $\mu$ be the dwell time of the switching law $\sigma=$ $\left(\left(t_{1}, i_{1}\right), \ldots,\left(t_{k}, i_{k}\right), \ldots\right)$, and $\delta>0$ is a constant. If $\mu \geq$ $\max \{\tau,(\ln (M(\delta+\widetilde{D}) / \delta)) /(\alpha-2 P M L)\}$, then $\varphi \in B_{\delta}\left(x^{i_{0}}\right)$ implies that the solution $x(t, 0, \varphi, \sigma)$ of (32) satisfies $x_{t_{k}} \in$ $B_{\delta}\left(x^{i_{k}}\right)$.

Theorem 8. Let $\mu$ be the dwell time of the switching law $\sigma=\left(\left(t_{1}, i_{1}\right), \ldots,\left(t_{k}, i_{k}\right), \ldots\right)$, and $\delta>0$ is a constant. If $\mu \geq \max \{\tau,(\ln (M(\delta+\widetilde{D}) / \delta)) /(\alpha-2 P M L)\}$, then $\varphi \in B_{\delta}\left(x^{i_{0}}\right)$ implies that the solution $x(t, 0, \varphi, \sigma)$ of (32) satisfies $x(t) \in$ $B_{M \delta}\left(\Gamma^{\prime}\right)$ for all $t \in R^{+}$.

\section{Attractiveness}

In this section, we first give the concept of an attractor of the switched system with switching law, and then we study the existence of the attractor of the nonlinear switched delay system (1). We will establish sufficient conditions which guarantee the existence of the attractor and furthermore we can find out the switching law and the attractor of the switching delay system (1).

Definition 9. A set $\Omega \subset R^{n}$ is called an attractor of the switched system (1) with a switching law $\sigma$, if there exists a $\delta>0$ such that, for any $\varepsilon>0$, there exists a $T>0$, such that, for any $\varphi \in B_{\delta}(\Omega) \cap C_{\tau}, t \geq T$ implies $\rho(x(t, 0, \varphi, \sigma), \Omega) \leq \varepsilon$.

Remark 10. According to Definition $9, \Omega$ is an attractor of the switched system (1) with a switching law $\sigma$ if and only if there exists $\delta>0$ such that $\varphi \in B_{\delta}(\Omega)$ implies

$$
\lim _{t \rightarrow \infty} x(t, 0, \varphi, \sigma)=0 .
$$

In this section, we will prove that $\Gamma$ is the attractor of system (1). For convenience, we first show that

$$
\varphi \in B_{\delta}(\Gamma) \Longrightarrow \lim _{t \rightarrow \infty} x(t, 0, \varphi, \sigma)=0
$$

is equivalent to

$$
\varphi \in B_{\delta}\left(x^{i}\right) \Longrightarrow \lim _{t \rightarrow \infty} x(t, 0, \varphi, \sigma)=0 .
$$

Lemma 11. For any given $\varepsilon>0, \lambda>0$, there exists $T(\varepsilon, \lambda)=$ $\ln (M \Delta / \lambda \varepsilon) /(\alpha-M L)>0$ such that if $\varphi \in B_{\varepsilon}(\Gamma), \varphi \in C_{\tau}$, then the solution $x_{i}(t, 0, \varphi)$ of the ith subsystem of (1) satisfies $x_{i t} \in B_{\lambda \varepsilon}\left(x^{i}\right), t \geq T$, where $\Delta \triangleq \sup \left\{\|x-y\|, x, y \in B_{\varepsilon}(\Gamma)\right\}$., If $\varphi \in B_{\varepsilon}\left(x^{j}\right)$ and $\varphi \in C_{\tau}$ especially, then one can take $T(\varepsilon, \lambda)=$ $\ln (M(\varepsilon+\widetilde{D}) / \lambda \varepsilon) /(\alpha-M L)>0$ such that when $t \geq T$ implies the solution of the ith subsystem of $(1) x_{i}(t, 0, \varphi)$ satisfies $x_{i t} \in$ $B_{\lambda \varepsilon}\left(x^{i}\right)$.

Proof. Let $x_{i}(t, 0, \varphi) \triangleq x_{i}(t)$ be the solution of the $i$ th subsystem of (1) and $\varphi \in B_{\varepsilon}(\Gamma)$, and then

$$
\begin{aligned}
& \left\|x_{i}(t)-x^{i}\right\| \\
& =\| e^{A_{i} t} \varphi(0)+\int_{0}^{t} e^{A_{i}(t-u)} f_{i}\left(x_{i}(u-\tau)\right) d u \\
& \quad-e^{A_{i} t} x^{i}-\int_{0}^{t} e^{A_{i}(t-u)} f_{i}\left(x^{i}\right) d u \| \\
& \leq\left\|e^{A_{i} t}\left(\varphi(0)-x^{i}\right)\right\| \\
& \quad+\left\|\int_{0}^{t} e^{A_{i}(t-u)}\left[f_{i}\left(x_{i}(u-\tau)\right)-f_{i}\left(x^{i}\right)\right] d u\right\| \\
& \leq M e^{-\alpha t}\left\|\varphi(0)-x^{i}\right\| \\
& \quad+\int_{0}^{t} M L e^{-\alpha(t-u)}\left\|x_{i}(u-\tau)-x^{i}\right\| d u,
\end{aligned}
$$

which implies that

$$
e^{\alpha t}\left\|x_{i t}-x^{i}\right\|_{\tau} \leq M\left\|\varphi-x^{i}\right\|_{\tau}+M L \int_{0}^{t} e^{\alpha u}\left\|x_{i u}-x^{i}\right\|_{\tau} d u
$$

and thus

$$
\left\|x_{i t}-x^{i}\right\|_{\tau} \leq M \Delta e^{-(\alpha-M L) t} .
$$

So, when $t>T=\ln (M \Delta / \lambda \varepsilon) /(\alpha-M L)$, we have

$$
\left\|x_{i t}-x^{i}\right\|_{\tau} \leq \lambda \varepsilon \text { and thus } x_{i t} \in B_{\lambda \varepsilon}\left(x^{i}\right) .
$$

If $\varphi \in B_{\varepsilon}\left(x^{j}\right)$ specially, it follows from (37), that

$$
\begin{aligned}
e^{\alpha t}\left\|x_{i t}-x^{i}\right\|_{\tau} \\
\leq M\left\|\varphi-x^{i}\right\|_{\tau}+M L \int_{0}^{t} e^{\alpha u}\left\|x_{i u}-x^{i}\right\|_{\tau} d u \\
\leq M\left[\left\|\varphi-x^{j}\right\|_{\tau}+\left\|x^{j}-x^{i}\right\|\right] \\
\quad+M L \int_{0}^{t} e^{\alpha u}\left\|x_{i u}-x^{i}\right\|_{\tau} d u \\
\leq M[\varepsilon+\widetilde{D}]+M L \int_{0}^{t} e^{\alpha u}\left\|x_{i u}-x^{i}\right\|_{\tau} d u,
\end{aligned}
$$


which implies that

$$
\left\|x_{i t}-x^{i}\right\|_{\tau} \leq M[\varepsilon+\widetilde{D}] e^{-(\alpha-M L) t}
$$

and thus, when $t \geq T=\ln (M(\varepsilon+\widetilde{D}) / \lambda \varepsilon) /(\alpha-M L)$, we have $\left\|x_{i t}-x^{i}\right\|_{\tau} \leq \lambda \varepsilon$; that is, $x_{i t} \in B_{\lambda \varepsilon}\left(x^{i}\right)$. This completes the proof.

Theorem 12. Suppose that the dwell time $\mu$ of the switching law $\sigma=\left\{\left(t_{1}, i_{1}\right),\left(t_{2}, i_{2}\right), \ldots\right\}$ satisfies $t_{1} \geq \ln (M \Delta / \lambda \varepsilon) /(\alpha-M L)$. If $\mu \geq \max \{\tau, \ln (M \Delta / \lambda \varepsilon) /(\alpha-M L)\}$, then the set $\Gamma$ is an attractor of the switched delay system (1).

Proof. From Lemma 11, we can see that we only need to prove that, for any $\varphi \in C_{\tau}, \varphi \in B_{1}\left(\cup_{i=1}^{N} x^{i}\right)$, the solution $x(t, 0, \varphi, \sigma)$ satisfies $\lim _{t \rightarrow \infty} \rho(x(t, 0, \varphi, \sigma),, \Gamma)=0$. In fact, we take $\varepsilon=1$ and $\lambda=1 / 2$ in Lemma 11, and then $\varphi \in C_{\tau}$ and $\varphi \in B_{1}\left(x^{i_{0}}\right)\left(i_{0} \in \mathbb{Q}\right)$ implies that $x_{t_{k}} \in B_{1 / 2^{k}}\left(x^{i_{k}}\right)$ if $\mu \geq \ln [2 M(1+\widetilde{D})] /(\alpha-M L), k=1,2, \ldots$ On the other hand, for $t \in\left[0, t_{1}\right]$, we have

$$
\begin{aligned}
& \rho(x(t, 0, \varphi, \sigma), \Gamma) \\
& =\rho\left(x_{i_{1}}(t, 0, \varphi), \Gamma\right) \triangleq \rho\left(x_{i_{1}}(t), \Gamma\right) \\
& =\inf \left\{\left\|x_{i_{1}}(t)-y\right\|, y \in \Gamma\right\} \\
& \leq\left\|x_{i_{1}}(t)-x_{i_{1}}\left(t, 0, x^{i_{0}}\right)\right\| \\
& =\left\|x_{i_{1}}(t)-\tilde{x}_{i_{1}}^{i_{0}}(t)\right\| \\
& =\| e^{A_{i_{1}} t} \varphi(0)+\int_{0}^{t} e^{A_{i_{1}}(t-u)} f_{i_{1}}\left(x_{i_{1}}(u-\tau)\right) d u \\
& -e^{A_{i_{1}} t} x^{i_{0}}-\int_{0}^{t} e^{A_{i_{1}}(t-u)} f_{i_{1}}\left(\tilde{x}_{i_{1}}^{i_{0}}(u-\tau)\right) d u \| \\
& \leq\left\|e^{A_{i_{1}} t}\left(\varphi(0)-x^{i_{0}}\right)\right\| \\
& +\left\|\int_{0}^{t} e^{A_{i_{1}}(t-u)}\left[f_{i_{1}}\left(x_{i_{1}}(u-\tau)\right)-f_{i_{1}}\left(\tilde{x}_{i_{1}}^{i_{0}}(u-\tau)\right)\right] d u\right\| \\
& \leq M e^{-\alpha t}\left\|\varphi(0)-x^{i_{0}}\right\| \\
& +\int_{0}^{t} M L e^{-\alpha(t-u)}\left\|x_{i_{1}}(u-\tau)-\widetilde{x}_{i_{1}}^{i_{0}}(u-\tau)\right\| d u,
\end{aligned}
$$

which implies that

$$
\begin{aligned}
& e^{\alpha t}\left\|x_{i_{1}}(t)-\tilde{x}_{i_{1}}^{i_{0}}(t)\right\| \\
& \quad \leq M\left\|\varphi-x^{i_{0}}\right\|_{\tau}+\int_{0}^{t} M L e^{\alpha u}\left\|x_{i_{1} u}-\tilde{x}_{i_{1} u}^{i_{0}}\right\|_{\tau} d u .
\end{aligned}
$$

Since the right hand side of the above inequality is an increasing function, we have

$$
\begin{aligned}
& e^{\alpha t}\left\|x_{i_{1} t}-\tilde{x}_{i_{1} t}^{i_{0}}\right\|_{\tau} \\
& \leq M\left\|\varphi-x^{i_{0}}\right\|_{\tau}+\int_{0}^{t} M L e^{\alpha u}\left\|x_{i_{1} u}-\tilde{x}_{i_{1} u}^{i_{0} u}\right\|_{\tau} d u, \\
& \left\|x_{i_{1} t}-\tilde{x}_{i_{1} t}^{i_{0}}\right\|_{\tau} \leq M\left\|\varphi-x^{i_{0}}\right\|_{\tau} e^{-(\alpha-M L) t},
\end{aligned}
$$

and thus

$$
\rho(x(t, 0, \varphi, \sigma), \Gamma) \leq\left\|x_{i_{1}}(t)-\tilde{x}_{i_{1}}(t)\right\| \leq M,
$$

since $\varphi \in B_{1}\left(x^{i_{0}}\right)$. When $t \in\left[t_{1}, t_{2}\right]$, since $x_{t_{1}} \in B_{1 / 2}\left(x^{i_{1}}\right)$, we have

$$
\begin{aligned}
& \rho(x(t, 0, \varphi, \sigma), \Gamma) \\
& =\rho\left(x_{i_{2}}\left(t-t_{1}, 0, x_{t_{1}}\right), \Gamma\right) \triangleq \rho\left(x_{i_{2}}\left(t-t_{1}\right), \Gamma\right) \\
& =\inf \left\{\left\|x_{i_{2}}\left(t-t_{1}\right)-y\right\|, y \in \Gamma\right\} \\
& \leq\left\|x_{i_{2}}\left(t-t_{1}\right)-x_{i_{2}}\left(t-t_{1}, 0, x^{i_{1}}\right)\right\| \\
& =\left\|x_{i_{2}}\left(t-t_{1}\right)-\tilde{x}_{i_{2}}^{i_{1}}\left(t-t_{1}\right)\right\| \\
& =\| e^{A_{i_{2}}\left(t-t_{1}\right)} x_{t_{1}}(0) \\
& +\int_{0}^{t-t_{1}} e^{A_{i_{2}}\left(t-t_{1}-u\right)} f_{i_{2}}\left(x_{i_{2}}(u-\tau)\right) d u \\
& -e^{A_{i_{2}} t} x^{i_{1}}-\int_{0}^{t-t_{1}} e^{A_{i_{2}}\left(t-t_{1}-u\right)} f_{i_{2}}\left(\tilde{x}_{i_{2}}^{i_{1}}(u-\tau)\right) d u \| \\
& \leq\left\|e^{A_{i_{2}}\left(t-t_{1}\right)}\left(x_{t_{1}}(0)-x^{i_{1}}\right)\right\| \\
& +\| \int_{0}^{t-t_{1}} e^{A_{i_{2}}\left(t-t_{1}-u\right)} \\
& \times\left[f_{i_{2}}\left(x_{i_{2}}(u-\tau)\right)-f_{i_{2}}\left(\tilde{x}_{i_{2}}^{i_{1}}(u-\tau)\right)\right] d u \| \\
& \leq M e^{-\alpha\left(t-t_{1}\right)}\left\|x_{t_{1}}(0)-x^{i_{1}}\right\| \\
& +\int_{0}^{t-t_{1}} M L e^{-\alpha\left(t-t_{1}-u\right)}\left\|x_{i_{2}}(u-\tau)-\widetilde{x}_{i_{2}}^{i_{1}}(u-\tau)\right\| d u,
\end{aligned}
$$

which implies that

$$
\begin{aligned}
& e^{\alpha\left(t-t_{1}\right)}\left\|x_{i_{2} t}-\tilde{x}_{i_{2} t}^{i_{1}}\right\|_{\tau} \\
& \leq M\left\|x_{t_{1}}-x^{i_{1}}\right\|_{\tau}+\int_{0}^{t-t_{1}} M L e^{\alpha u}\left\|x_{i_{2} u}-\tilde{x}_{i_{2} u}^{i_{1}}\right\| \|_{\tau} d u, \\
& \left\|x_{i_{2} t}-\tilde{x}_{i_{2} t}^{i_{1}}\right\|_{\tau} \leq M\left\|x_{t_{1}}-x^{i_{1}}\right\|_{\tau} e^{-(\alpha-M L)\left(t-t_{1}\right)},
\end{aligned}
$$

and thus

$$
\rho(x(t, 0, \varphi, \sigma), \Gamma) \leq\left\|x_{i_{2} t}-\tilde{x}_{i_{2} t}^{i_{1}}\right\|_{\tau} \leq \frac{1}{2} M,
$$


since $x_{t_{1}} \in B_{1 / 2}\left(x^{i_{1}}\right)$. Suppose that, for $t \in\left[t_{k-1}, t_{k}\right]$,

$$
\rho(x(t, 0, \varphi, \sigma), \Gamma) \leq \frac{1}{2^{k-1}} M
$$

When $t \in\left[t_{k}, t_{k+1}\right]$, since $x_{t_{k}} \in B_{1 / 2^{k}}\left(x^{i_{k}}\right)$, (take $\varepsilon=1 / 2^{k}$ in Lemma 11), we have

$$
\begin{aligned}
\rho(x & (t, 0, \varphi, \sigma), \Gamma) \\
= & \rho\left(x_{i_{k+1}}\left(t-t_{k}, 0, x_{t_{k}}\right), \Gamma\right) \\
= & \rho\left(x_{i_{k+1}}\left(t-t_{k}\right), \Gamma\right) \\
= & \inf \left\{\left\|x_{i_{k+1}}\left(t-t_{k}\right)-y\right\|, y \in \Gamma\right\} \\
\leq & \left\|x_{i_{k+1}}\left(t-t_{k}\right)-x_{i_{k+1}}\left(t-t_{k}, 0, x^{i_{k}}\right)\right\| \\
\triangleq & \left\|x_{i_{k+1}}\left(t-t_{k}\right)-\tilde{x}_{i_{k+1}}^{i_{k}}\left(t-t_{k}\right)\right\| \\
= & \| e^{A_{i_{k+1}}\left(t-t_{k}\right)} x_{t_{k}}(0) \\
& \quad+\int_{0}^{t-t_{k}} e^{A_{i_{k+1}}\left(t-t_{k}-u\right)} f_{i_{k+1}}\left(x_{i_{k+1}}(u-\tau)\right) d u \\
& \quad-e^{A_{i_{k+1}}\left(t-t_{k}\right)} x^{i_{k}} \\
& \quad-\int_{0}^{t-t_{k}} e^{A_{i_{k+1}}\left(t-t_{k}-u\right)} f_{i_{k+1}}\left(\tilde{x}_{i_{k+1}}^{i_{k}}(u-\tau)\right) d u \| \\
\leq & M e^{-\alpha\left(t-t_{k}\right)}\left\|x_{t_{k}}(0)-x^{i_{k}}\right\| \\
+ & M L \int_{0}^{t-t_{k}} e^{-\alpha\left(t-t_{k}-u\right)}\left\|x_{i_{k+1}}(u-\tau)-\tilde{x}_{i_{k+1}}^{i_{k}}(u-\tau)\right\| d u \\
&
\end{aligned}
$$

which implies that

$$
\left\|x_{i_{k+1} t}-\tilde{x}_{i_{k+1} t}^{i_{k}}\right\|_{\tau} \leq M\left\|x_{t_{k}}-x^{i_{k}}\right\|_{\tau} e^{-(\alpha-M L)\left(t-t_{1}\right)} \leq \frac{1}{2^{k}} M
$$

and thus

$$
\rho(x(t, 0, \varphi, \sigma), \Gamma) \leq \frac{1}{2^{k}} M, \quad t \in\left[t_{k}, t_{k+1}\right] .
$$

Therefore, we have

$$
\lim _{t \rightarrow \infty} \rho(x(t, 0, \varphi, \sigma), \Gamma)=0,
$$

which means that $\Gamma$ is the attractor of system (1) with a switching law $\sigma$.

Similarly, we can study the existence of the attractor of the switched delay system (32) with the assumptions (H1)'-(H3)' and obtain the following results.

Lemma 13. For any given $\varepsilon>0, \lambda>0$, there exists

$$
T(\varepsilon, \lambda)=\max \left\{\tau, \frac{\ln (M(\delta+\Delta) / \delta)}{\alpha-2 P M L}\right\},
$$

such that if $\varphi \in B_{\varepsilon}\left(\Gamma^{\prime}\right)$ and $\varphi \in C_{\tau}$, then the solution $x_{i}(t, 0, \varphi)$ of the ith subsystem of (32) satisfies $x_{i t} \in B_{\lambda \varepsilon}\left(x^{i}\right), t \geq T$. If $\varphi \in B_{\varepsilon}\left(x^{j}\right)$ especially, then one can take $T(\varepsilon, \lambda)=\ln (M(\varepsilon+$ $\widetilde{D}) / \lambda \varepsilon) /(\alpha-2 P M L)>0$ such that when $t \geq T$ implies the solution $x_{i}(t, 0, \varphi)$ of the ith subsystem of (32) satisfies $x_{i t} \in$ $B_{\lambda \varepsilon}\left(x^{i}\right), t \geq T$.

Theorem 14. Suppose that the dwell time $\mu$ of the switching law $\sigma=\left\{\left(t_{1}, i_{1}\right),\left(t_{2}, i_{2}\right), \ldots\right\}$ satisfies $t_{1} \geq \ln (M \Delta / \lambda \varepsilon) /(\alpha-2 P M L) ;$ if $\mu \geq \max \{\tau, \ln (M \Delta / \lambda \varepsilon) /(\alpha-2 P M L)\}$, then the set $\Gamma^{\prime}$ is an attractor of the switched delay system (32).

\section{Acknowledgments}

This work was partially supported by the NSFC (nos. 11071257, 61290325, and 11171019), the ARC Discovery Projects, HUST Startup Research Fund, HUST Independent Innovation Research Fund (GF and Natural Science 2013), the Natural Science Foundation of Beijing (no. 1122009), and the Grant of Beijing Education Committee Key Project (no. KZ201310028031). General Program of Science and Technology Development Project of Beijing Municipal Education Commission (no. KM200810028001), and Beijing Municipal Commission of Education (KM2014).

\section{References}

[1] M. S. Branicky, Studies in hybrid systems: modeling, analysis, control [ScD Thesis], MIT EECS, 1995.

[2] Z. Li, Y. Soh, and C. Wen, Switched and Impulsive Systems, vol. 313 of Lecture Notes in Control and Information Sciences, Springer, Berlin, Germany, 2005.

[3] D. Liberzon, Switching in Systems and Control, Systems \& Control: Foundations \& Applications, Birkhäuser, Boston, Mass, USA, 2003.

[4] W. P. Dayawansa and C. F. Martin, "A converse Lyapunov theorem for a class of dynamical systems which undergo switching," IEEE Transactions on Automatic Control, vol. 44, no. 4, pp. 751-760, 1999.

[5] M. A. Wick, P. Peleties, and R. A. Decarlo, "Switched controller synthesis for the quadratic stabilization of a pair of unstable linear systems," European Journal of Control, vol. 4, pp. 140-147, 1998.

[6] R. N. Shorten and K. S. Naredra, "On the stability and existence of common Lyapunov functions for stable linear switching systems," in Proceedings of the IEEE Conference on Decision and Control, vol. 4, pp. 3723-3724, 1998.

[7] D. Liberzon, J. P. Hespanha, and A. S. Morse, "Stability of switched systems: a Lie-algebraic condition," Systems \& Control Letters, vol. 37, no. 3, pp. 117-122, 1999.

[8] H. Xu and K. L. Teo, "Exponential stability with $L_{2}$-gain condition of nonlinear impulsive switched systems," Institute of Electrical and Electronics Engineers, vol. 55, no. 10, pp. 24292433, 2010.

[9] J. Liu, X. Liu, and W.-C. Xie, "Generalized invariance principles for switched delay systems," IMA Journal of Mathematical Control and Information, vol. 28, no. 1, pp. 19-39, 2011.

[10] X. Liu, "Stabilization of switched linear systems with modedependent time-varying delays," Applied Mathematics and Computation, vol. 216, no. 9, pp. 2581-2586, 2010. 
[11] M. S. Branicky, "Multiple Lyapunov functions and other analysis tools for switched and hybrid systems," IEEE Transactions on Automatic Control, vol. 43, no. 4, pp. 475-482, 1998.

[12] S. Pettersson and B. Lennartson, "Stability and robustness for hybrid systems," in Proceedings of the IEEE Conference on Decision and Control, vol. 12, pp. 1202-1207, 1996.

[13] D. Boularas and D. Cheban, "Asymptotic stability of switching systems," Electronic Journal of Differential Equations, vol. 2010, no. 21, pp. 1-18, 2010.

[14] J. Liu, X. Liu, and W.-C. Xie, "Input-to-state stability of impulsive and switching hybrid systems with time-delay," Automatica, vol. 47, no. 5, pp. 899-908, 2011.

[15] V. Lakshmikantham, S. Leela, and A. A. Martynyuk, Practical Stability of Nonlinear Systems, World Scientific, Singapore, 1990.

[16] J. LaSalle and S. Lefschetz, Stability by Liapunov's Direct Method, with Applications, vol. 4 of Mathematics in Science and Engineering, Academic Press, New York, NY, USA, 1961.

[17] X. Xu and G. Zhai, "Practical stability and stabilization of hybrid and switched systems," IEEE Transactions on Automatic Control, vol. 50, no. 11, pp. 1897-1903, 2005.

[18] J. Leth and R. Wisniewski, "On formalism and stability of switched systems," Journal of Control Theory and Applications, no. 1, pp. 123-130, 2011.

[19] B. Du and X. Zhang, "Delay-dependent stability analysis and synthesis for uncertain impulsive switched system with mixed delays," Discrete Dynamics in Nature and Society, vol. 2011, Article ID 381571, 9 pages, 2011.

[20] M. Y. Shieh, J. S. Chiou, and C. M. Cheng, "Delay independence stability analysis and switching law design for the switched time-delay systems," Information Technology Journal, vol. 10, no. 6, pp. 1201-1207, 2011.

[21] X. Xu and P. J. Antsaklis, "Practical stabilization of integrator switched systems," in Proceedings of the American Control Conference, pp. 2767-2772, Denver, Colo, USA, June 2003.

[22] X. Xu, G. Zhai, and S. He, "On practical asymptotic stabilizability of switched affine systems," Nonlinear Analysis: Hybrid Systems, vol. 2, no. 1, pp. 196-208, 2008. 


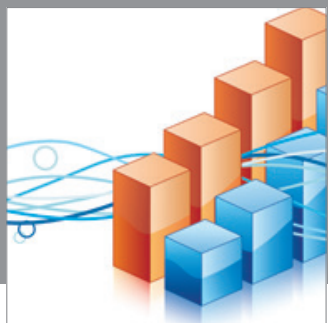

Advances in

Operations Research

mansans

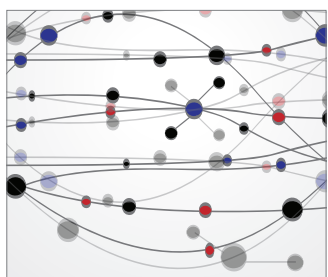

The Scientific World Journal
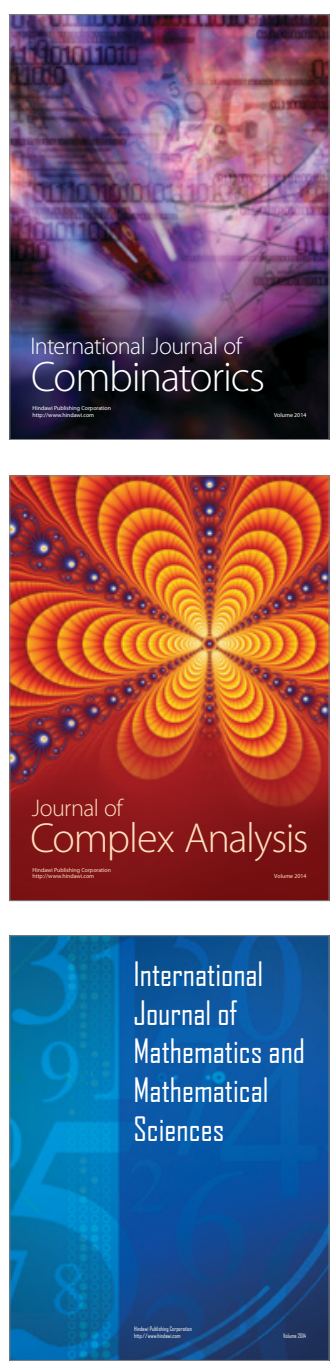
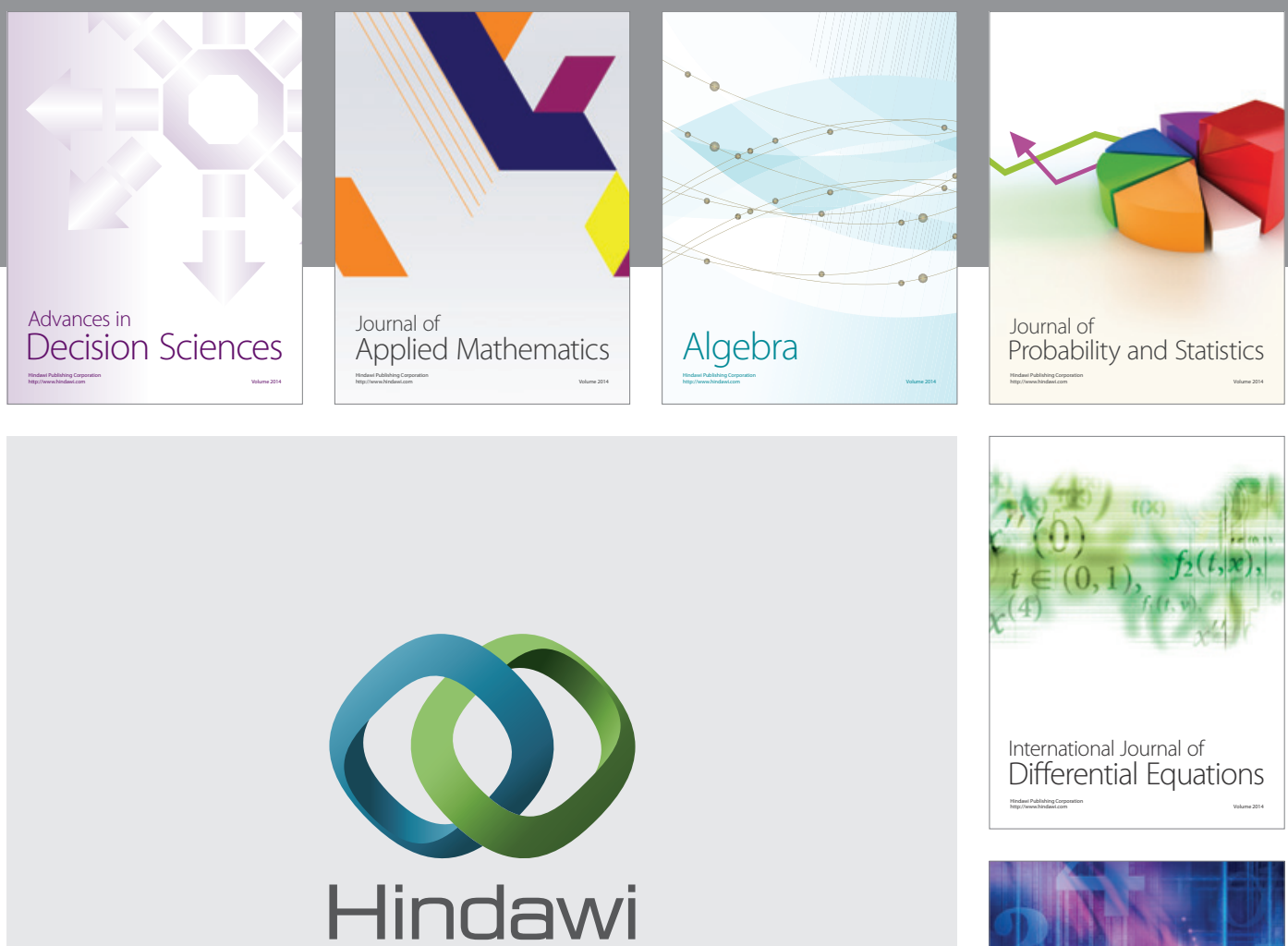

Submit your manuscripts at http://www.hindawi.com
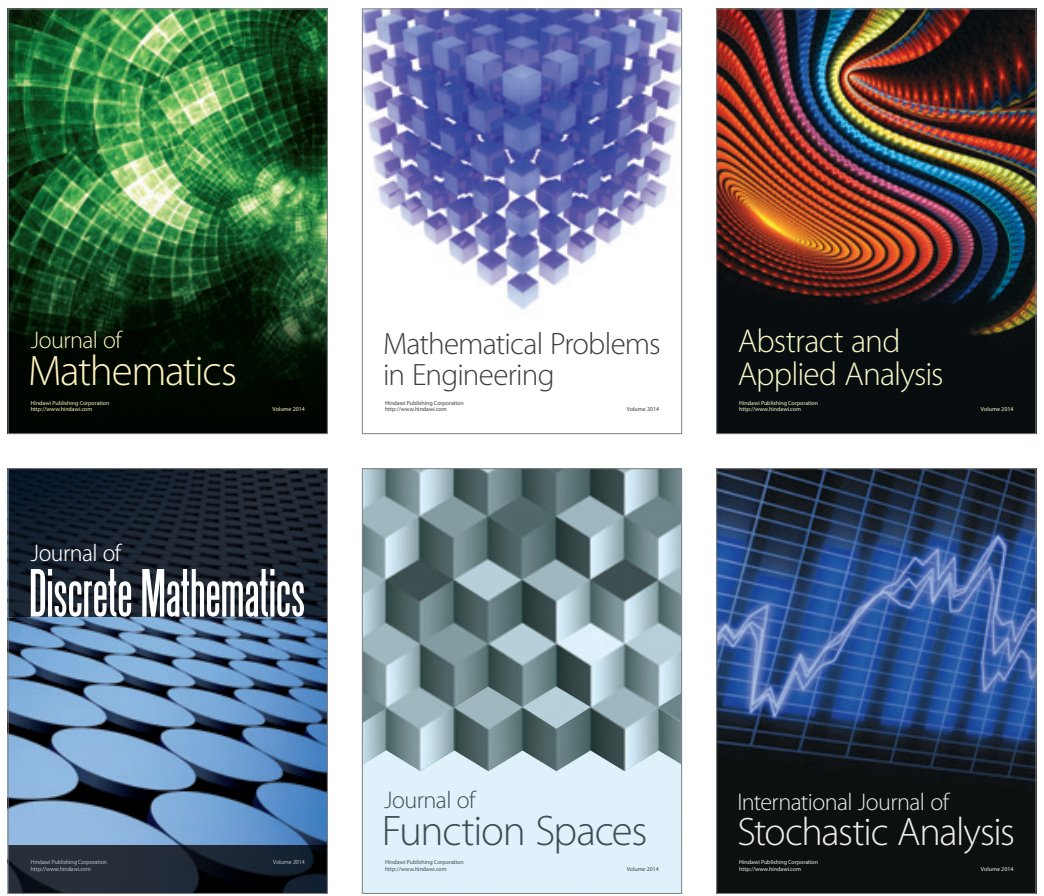

Journal of

Function Spaces

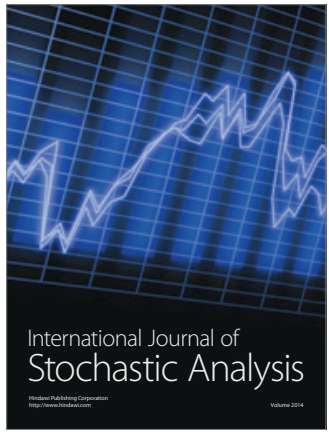

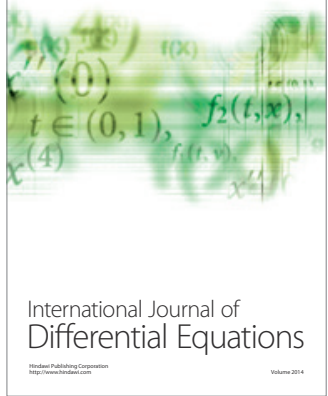
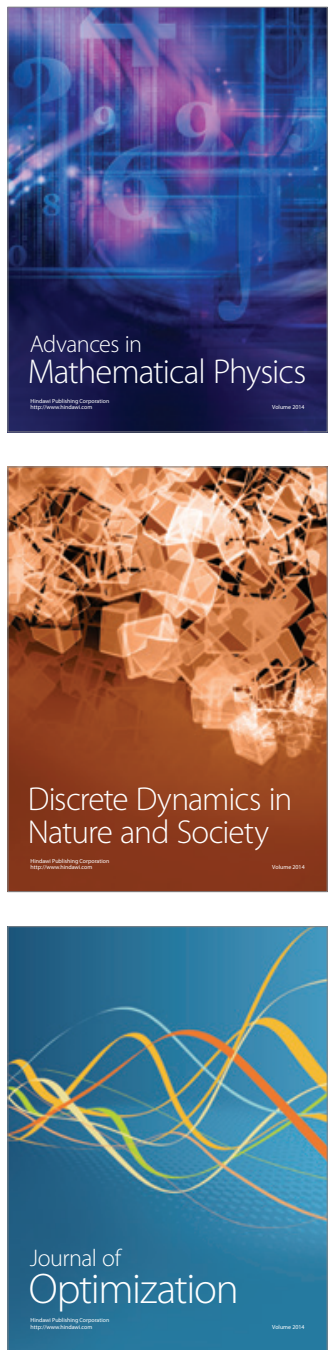